\title{
Nosocomial spread of OXA-232-producing Klebsiella pneumoniae ST15 in a teaching hospital, Shanghai, China
}

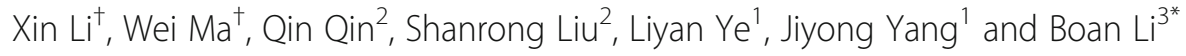

\begin{abstract}
Background: The spread and outbreak of Enterobacteriaceae producing OXA-48-like carbapenemases have become more and more prevalent in China.

Results: A total of 62 non-duplicated OXA-232-producing K. pneumoniae (OXA232Kp) were isolated between 2015 and 2017. An outbreak of OXA232Kp was observed in burn ICU. The 62 OXA232Kp isolates were all belongs to ST15 and categorized into two PFGE types (A and B). Type A was dominated of the isolates, which contained 61 clinical isolates and divided into 10 subtypes (A1-A10). In addition, most of OXA232Kp strains exhibited low-level carbapenems resistance. All strains carried a $6141 \mathrm{bp}$ CoIKP3 plasmid harboring the bla OXA-232 gene which is highly homologous to other bla OXA-232-bearing plasmids involved in other studies in eastern China.

Conclusions: In this study, clone transmission of OXA232Kp ST15was observed. Highly significant homology among the bla $a_{\mathrm{OXA}-232}$-bearing plasmids indicated the important role of the $6.1 \mathrm{~kb}$ ColE-like plasmid on the prevalence of bla $\mathrm{OXA}-232$ gene in China.
\end{abstract}

Keywords: Outbreak, Klebsiella pneumoniae, OXA-232, Sequence type

\section{Background}

The most alarming resistance trends are those observed for Enterobacteriaceae, and of particular concern has been the emergence of resistance to carbapenem antimicrobial drugs among Enterobacteriaceae. Carbapenem resistance in Enterobacteriaceae is usually attributed to the production of various carbapenemases, a set of enzymes that lead to the hydrolysis of beta-lactams [1]. OXA-48, a novel class D carbapenemase, was identified in a clinical Klebsiella pneumoniae isolate in 2004 [2]. Since then, OXA-48producing Enterobacteriaceae has been reported worldwide [3]. Up until now, OXA-48 and its several variants have been identified in Enterobacteriaceae. These variants are one to five amino acid substitutions differs from OXA-48 [3, 4]. Most of OXA-48-type carbapenemases hydrolyze carbapenems slightly, while some OXA-48-like variants (such as OXA-163, OXA-247 and OXA-405) only

\footnotetext{
* Correspondence: Iba@263.net

${ }^{+}$Xin Li and Wei Ma contributed equally to this work.

${ }^{3}$ Center for Clinical Laboratory, the 302 Hospital of Chinese PLA, Beijing 100039, China

Full list of author information is available at the end of the article
}

hydrolyze expanded-spectrum cephalosporins but not carbapenems [4]. However, associated with the impaired permeability or the production of extended-spectrum $\beta$ lactamases, some OXA-48-like enzymes can lead to highlevel carbapenems resistance [5].

OXA-48 was mainly detected in K. pneumoniae. Diverse sequence types (STs) of dominant OXA-48-producing $K$. pneumoniae have been identified in outbreaks or solitary case reports worldwide [3]. ST11 and ST116 were present in Taiwan, China [6]. An outbreak of OXA-48-producing K. pneumoniae ST147 and ST383 has been reported [7], while other clones, such as ST37 and ST307, have also been identified sporadically [8]. The $b l a_{\text {OXA-48 }}$ gene is mainly found on an IncL/M-type self-transferable plasmid of approximately $62 \mathrm{~kb}$, and flanked by two IS1999 elements to form a functional composite transposon Tn1999, which does not carry any other antibiotic resistance gene $[2,5]$. In addition, several bla ${ }_{\text {OXA-48-bearing Tn1999-like }}$ transposon derivatives ( $\operatorname{Tn} 1999.2$ to $\operatorname{Tn} 1999.5)$ were also discovered [3].

OXA-232, an OXA-48-like variant, was identified in France from a K. pneumoniae isolate in 2013. OXA-232 
differs one (at Arg214Ser) and five amino acid substitutions from OXA-181 and was considered to be a mutant derivative of OXA-181 and not from OXA-48 [9]. Similar to OXA-48, OXA-232 has a reduced ability to hydrolyze carbapenems but exhibits higher hydrolysis activity against penicillins [9]. Since then, OXA-232-producing K. pneumoniae (OXA232Kp) had been identified worldwide. These OXA232Kp isolates belong to ST14, ST15, ST16, ST17, ST147, ST231, ST307, and ST395 [10-18]. Almost all OXA232Kp isolates had plasmids with more than $99 \%$ sequence identity to pOXA-232, and had a size of $6.1-\mathrm{kb}$ and carried the ColE-type backbone [9]. All these ColEtype plasmids carried the mobilization system (MobA-D), a replication gene $($ rep $A)$, truncated parts of erythromycin esterase $(\triangle e r e A)$ and the transcriptional regulator $(\triangle l y s R)$ [19]. The sequence of pOXA-232 was identical to that of plasmid pKP3-A bearing the bla $a_{\text {OXA-181 }}$ gene [20], except for almost entire deletion of the ISEcp1 transposase gene in pOXA-232.

OXA232Kp has been reported in eastern China. All these OXA232Kp isolates belong to ST15 and carry a 6.1-kp ColKP3 plasmid bearing the bla $a_{\mathrm{OXA}-232}$ gene [2123]. In this study, we reported a nosocomial outbreak of OXA232Kp in a teaching hospital in Shanghai involved with 61 patients. The phenotypic and genotypic characteristics of OXA232Kp isolates were analyzed.

\section{Methods}

\section{Bacterial strains}

All K. pneumoniae isolates recovered from various clinical specimens between 2015 and 2017 at Changhai hospital in Shanghai, China were collected and further identified by VITEK $^{\oplus}$ MS (bioMérieux SA, Marcy-l'Etoile, France). The common carbapenemase genes including $b l a_{\mathrm{KPC}}, b l a_{\mathrm{NDM}}$, $b l a_{\mathrm{OXA}-48}$ and $b l a_{\mathrm{IMP}}$ from isolates that exhibited nonsusceptibility to carbapenems were screened by PCR amplification, as previously described [24]. E. coli ATCC 25922 was used as the quality control strain for antimicrobial susceptibility test. Salmonella ser. Braenderup strain (H9812) was used as a reference standard for PFGE.

\section{Antimicrobial susceptibility test}

The MICs of cefotaxime (CTX), ceftazidime (CAZ), piperacillin-tazobactam (TZP), imipenem (IMP), meropenem (MEM), ertapenem (ETP), amikacin (AK), ciprofloxacin (CIP), tigecycline (TGC), sulfamethoxazole/ trimethoprim (SXT) and polymyxin B (PB) were measured by broth microdilution method using Biofosun ${ }^{\odot}$ Gram-negative panels (Biofosun Biotech, Co., Ltd., Shanghai, China). Results were interpreted according to the interpretive standards of the Clinical Laboratory Standards Institute [25].

\section{PFGE and MLST}

PFGE with restriction enzyme XbaI was performed for all OXA232Kp isolates as previously described [26]. The PFGE patterns were analyzed by BioNumerics 7.6 software (Applied Maths NV, Sint-Martens-Latem, Belgium) using the dice similarity coefficient. Strains possessing $\geq 85 \%$ genetic similarity or fewer than four fragment differences in PFGE profiles were considered as the same clone (type). Strains (types) with pattern that differ from the original pattern by $\leq 3$ fragments differences were considered to be a subtype of the outbreak strain [27]. MLST was carried out according to the protocols provided on the MLST website (http://www.pasteur.fr/ recherche/genopole/PF8/mlst/Kpneumoniae).

\section{Resistance genes analysis}

Genome DNA was extracted from the clinical OXA232Kp isolates using the DNeasy ${ }^{\circ}$ UltraClean $^{\circledR}$ Microbial Kit (QIAGEN GmbH, 40,724 Hilden, Germany). Genome sequencing was performed with a paired-end library with an average insert size of $350 \mathrm{bp}$ on a HiSeq X Ten sequencer (Illumina, CA, USA). Then, de novo assembly of the filtered reads was performed with SPAdes 3.12 (http://cab. spbu.ru/software/spades/). Draft genome was assembled into scaffold. Acquired antimicrobial resistance genes and plasmid replicons were identified using the ResFinder and PlasmidFinder software available from the Center for Genomic Epidemiology (http://genomicepidemiology.org/).

\section{Results}

\section{Emergence and outbreak of OXA232Kp}

A total of 141 non-duplicated $K$. pneumoniae isolates that exhibited non-susceptibility to carbapenems were recovered from various clinical specimens between 2015 and 2017. Among these isolates, 62 (43.9\%) were OXA232Kp, while 60 (42.6\%) produced KPC. Among 62 strains of OXA232Kp, 31 (50\%) were recovered from sputum, 11, 11 and 5 isolates were recovered from wound secretion, urine and drainage sample, respectively. In July 2015, OXA232Kp first appeared at the burn ICU, followed by an outbreak in the hospital (Fig. 1). Among 62 patients with OXA232Kp isolates, 7 patients died, and 56 patients (90.3\%) showed improvement and discharged.

\section{PFGE and MLST analysis}

The 62 OXA232Kp isolates were categorized into two PFGE types (A and $\mathrm{B}$ ). The majority of the isolates belonged to types $\mathrm{A}$ which contained 61 clinical isolates and was divided into ten subtypes (A1-A10), while types $B$ was detected in only one isolate (Table 1). In July 2015, type A2 of OXA232Kp strain emerged in the hospital. This followed by an outbreak in the burn ICU in the next few months. Meanwhile, new OXA232Kp subtypes (A3-A10) emerged and were found spread to burn 


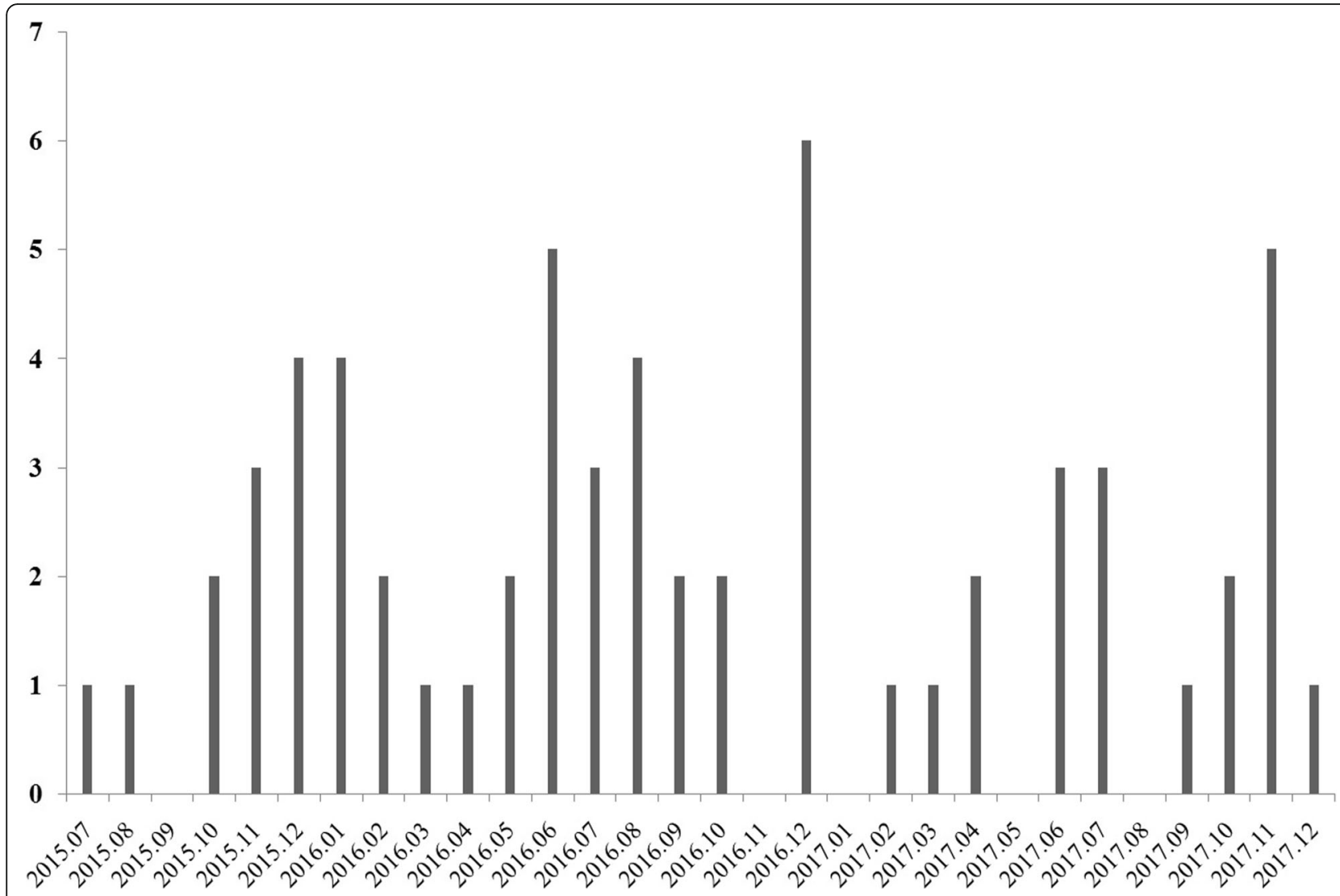

Fig. 1 The distribution of the OXA-232-producing K. pneumoniae isolates

ICU other wards (Fig. 1). MLST was performed in one representative of each PFGE types and subtypes. All strains belonged to ST15.

\section{Antimicrobial susceptibilities}

The antimicrobial susceptibility patterns were listed in Table 1. All strains presented resistance to third generation cephalosporins and their enzyme inhibitors mixture (CTX, CAZ and TZP), and exhibited heterogeneous carbapenem resistance patterns. However, they were susceptible to tigecycline and polymyxin B.

\section{Plasmid and resistance determinants}

Genome sequencing was performed in one representative strain of each PFGE types and subtypes. All the sequences were uploaded to GenBank with BioProject Accession PRJNA523565. All strains carried bla OXA-232 gene, which located in a $6141 \mathrm{bp}$ ColKP3 plasmid. In addition, other resistance determinants were also identified (Table 1).

\section{Discussion}

OXA-232 was first identified in France in 2013 from a $K$. pneumoniae isolate [9]. Since then, OXA232Kp have been discovered worldwide [10-18]. In this study, a nosocomial outbreak of epidemics OXA232Kp involving 61 patients was discovered in a teaching hospital in Shanghai, China. In this hospital, OXA232Kp acted as the main species of carbapenem-resistant $K$. pneumoniae between 2015 and 2017, which $43.9 \%$ were OXA-232 positive, while only $42.6 \%$ of them produced KPC. This is quite different from data obtained from other part of the world, where production of KPC, OXA-48, and metallo- $\beta$-lactamases such as NDM is the main mechanism of carbapenem-resistant $K$. pneumoniae [1]. In this study, OXA232Kp mainly disseminated in the burn ICU (Fig. 2) and appeared to be clonal. At the same time, several outbreak caused by OXA232Kp have been identified in the same area [21-23]. Recently, an outbreak of OXA-48-producing $K$. pneumoniae involving 34 patients at a respiratory ICU has been reported [7]. These results indicated that emergence of $K$. pneumoniae strains producing OXA-48-like carbapenemases have become increasingly frequent in China. It is crucial to recognize the impact of clonal dissemination during the prevalence of these pathogens and strengthen the surveillance and reporting system of carbapenem-resistant $K$. pneumoniae in hospital infection control measures.

In outbreaks of OXA232Kp worldwide, diverse sequence types have been identified, including ST14, ST15, ST16, ST17, ST147, ST231, ST307, and ST395. In Europe and some Asia countries, the majority of OXA232Kp strains 
Table 1 Phenotypic and genotypic characteristics of OXA-232-producing K. pneumoniae isolates

\begin{tabular}{|c|c|c|c|c|c|c|c|c|c|c|c|c|c|c|}
\hline \multirow{2}{*}{$\begin{array}{l}\text { PFGE } \\
\text { type }\end{array}$} & \multirow{2}{*}{$\begin{array}{l}\text { MLST } \\
\text { type }\end{array}$} & \multirow{2}{*}{$\begin{array}{l}\text { Isolates } \\
\text { number }\end{array}$} & \multirow[t]{2}{*}{ Resistance determinants } & \multicolumn{11}{|c|}{ Minimal inhibitory concentration (mg/L) } \\
\hline & & & & CTX & CAZ & PZT & IPM & MEM & ETP & AK & TGC & PB & SXT & CIP \\
\hline A1 & ST 15 & 36 & 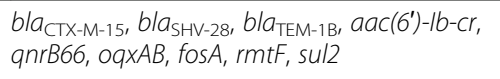 & $>64$ & $>32$ & $>256$ & 2 & 2 & 16 & $>128$ & $\leq 0.5$ & 1 & $>8$ & $>16$ \\
\hline $\mathrm{A} 2$ & ST 15 & 3 & 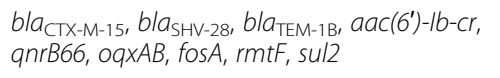 & $>64$ & $>32$ & $>256$ & 32 & 32 & 128 & $>128$ & $\leq 0.5$ & 1 & $>8$ & $>16$ \\
\hline A3 & ST 15 & 9 & 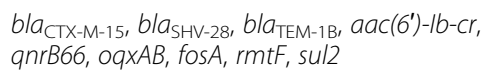 & $>64$ & $>32$ & $>256$ & 1 & 2 & 16 & $>128$ & $\leq 0.5$ & 1 & $>8$ & $>16$ \\
\hline $\mathrm{A} 4$ & ST 15 & 1 & $\begin{array}{l}\text { bla }_{\mathrm{CTX}-\mathrm{M}-15}, \text { bla } a_{\mathrm{SHV}-28,}, b l a_{\mathrm{TEM}-1 \mathrm{~B}}, \text { aac }\left(6^{\prime}\right)-1 b-c r, \\
\text { qniB66, oqxAB, fosA, rmtF, sul2 }\end{array}$ & $>64$ & $>32$ & $>256$ & 1 & 2 & 16 & $>128$ & $\leq 0.5$ & 1 & $>8$ & $>16$ \\
\hline A5 & ST 15 & 5 & $\begin{array}{l}\text { bla }_{\mathrm{CTX}-\mathrm{M}-15}, \text { bla } a_{\mathrm{SHV}-28,}, b l a_{\mathrm{TEM}-1 \mathrm{~B}}, \text { aac }\left(6^{\prime}\right)-1 b-c r, \\
\text { qniB66, oqxAB, fosA, rmtF, sul2 }\end{array}$ & $>64$ & $>32$ & $>256$ & 1 & 4 & 16 & $>128$ & $\leq 0.5$ & 1 & $>8$ & $>16$ \\
\hline A6 & ST 15 & 1 & 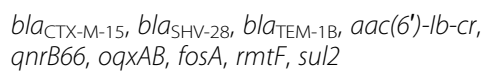 & $>64$ & $>32$ & $>256$ & 1 & 4 & 64 & $>128$ & $\leq 0.5$ & 1 & $>8$ & $>16$ \\
\hline A7 & ST 15 & 3 & 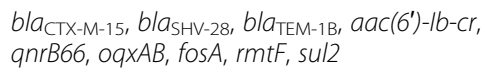 & $>64$ & $>32$ & $>256$ & 4 & 8 & 32 & $>128$ & $\leq 0.5$ & 1 & $>8$ & $>16$ \\
\hline A8 & ST 15 & 1 & 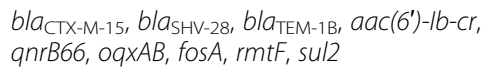 & $>64$ & $>32$ & $>256$ & 1 & 2 & 16 & $>128$ & $\leq 0.5$ & 1 & $>8$ & $>16$ \\
\hline A9 & ST 15 & 1 & $\begin{array}{l}\text { bla } \\
\text { CTX-M-15, bla } a_{\mathrm{SHV}-28,} \text {, bla } a_{\mathrm{TEM}-1 \mathrm{~B},} \text {, aac }\left(6^{\prime}\right)-1 b-\mathrm{cr}, \\
\text { anrB66, oqxAB, fosA, rmtF, sul2 }\end{array}$ & $>64$ & $>32$ & $>256$ & 64 & 64 & 256 & $>128$ & $\leq 0.5$ & 1 & $>8$ & $>16$ \\
\hline $\mathrm{A} 10$ & ST 15 & 1 & 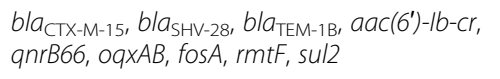 & $>64$ & $>32$ & $>256$ & 2 & 4 & 32 & $>128$ & $\leq 0.5$ & 1 & $>8$ & $>16$ \\
\hline B & ST 15 & 1 & 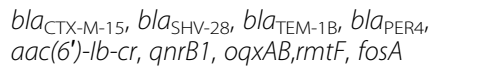 & $>64$ & $>32$ & $>256$ & 1 & 2 & 16 & $>128$ & $\leq 0.5$ & 1 & $>8$ & $>16$ \\
\hline
\end{tabular}

CTX: cefotaxime, CAZ: Ceftazidime, PZT: piperacillin-tazobactam, IMP: imipenem, MEM: meropenem, ETP: ertapenem, AK: amikacin, TGC: tigecycline, PB: polymyxin B, SXT: sulfamethoxazole/trimethoprim, CIP: ciprofloxacin

were ST14 and ST16 [10-18]. It has been confirmed that K. pneumoniae ST15 is the high-risk clones producing OXA-48, KPCs, CTX-M-15 and other ESBLs or carbapenemases. Outbreaks of OXA-48-producing K. pneumoniae ST15 have been reported worldwide [28-30]. OXA232Kp
ST15 has only sporadically presented in United Kingdom, but there has never been an outbreak [18]. However, all OXA232Kp strains belonged to ST15 in this study (Table 1). Meanwhile, other OXA232Kp strains isolated from eastern China were also ST15 [21-23], suggesting the

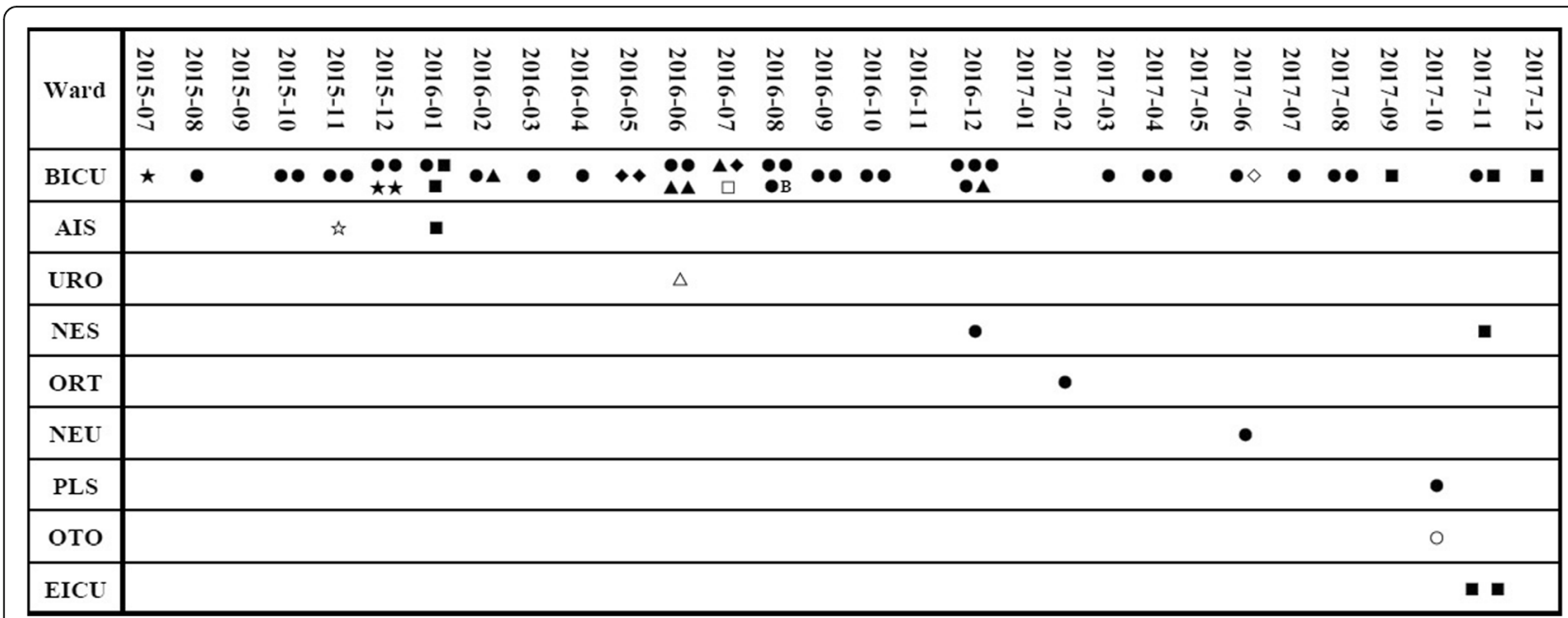

Fig. 2 The date, frequency and location of emergence of OXA232Kp strains with different PFGE types The horizontal coordinate shows the date on which OXA232Kp strains were recovered. The wards were listed in the longitudinal coordinate. BICU: Burn ICU; AIS: Department of anus \& intestine surgery; URO: Department of Urology; NES: Department of Neurosurgery; ORT: Department of Orthopedics; NEU: Department of Neurology; PLS Department of Plastic surgery; OTO: Department of Otolaryngology; EICU: Emergency ICU. Clinical isolates with different PFGE types are indicated by

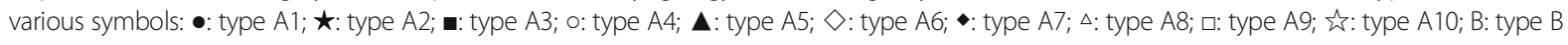


transmission and spread advantage of this clone in this region, despite the hypovirulence of this clone has been confirmed [23]. Further efforts are required to gain a better understanding about the mechanisms underlying differential prevalence.

According to their case records, $90.3 \%$ of patients showed improvement and discharged. Study has shown that OXA232Kp ST15 did not cause lethal infections in immunocompromised patients [23]. Another reason is that the low-level resistance of the stain to carbapenems. The majority of OXA232Kp exhibited low-level resistant or even susceptible to some carbapenems (Table 1). Animal studies and patient outcome data indicated that carbapenems retain meaningful in vitro activity against carbapenemase-producing $K$. pneumoniae isolates with low-level carbapenem resistance [31]. Therefore, timely and effective anti-infective treatment may be an important reason for good outcomes of patients with OXA232Kp infection. However, our data suggest that quinolones and aminoglycosides are no longer suitable for the treatment of infections caused by OXA232Kp (Table 1).Similar to OXA-48, OXA-232 has a reduced ability to hydrolyze carbapenems [9]. Despite the production of CTX-M-15, an enzyme that is considered to mediate high-level carbapenem-resistant phenotypes together with OXA-48like carbapenemases [5], few OXA232Kp isolates exhibited high-levels of carbapenem resistance (Table 1). Thus, what extent the impacts of ESBLs are on the carbapenem resistance of OXA232Kp is not clear yet. The weak hydrolytic ability of OXA-48-like to carbapenems may lead to an underestimation of the prevalence of clinical isolates producing this group of carbapenemases in China, because most clinical microbiology laboratories only screened the prevalence of carbapenemases among isolates with carbapenem-resistant phenotypes. Changes are needed in clinical microbiology analysis procedures to enhance phenotypic and molecular detection of OXA-48-like carbapenemases.

In this study, all strains carried a 6141 bp ColKP3 plasmid harboring the bla OXA-232 gene, which was $100 \%$ identical to the previously reported $b l a_{\mathrm{OXA}-232}$-bearing plasmid pOXA-232 and pkNICU5, with 100\% coverage $[9,21]$. Similar plasmid has also been detected in another city in the same region [23]. Except for almost entirely deletion of the ISEcp1 transposase gene, the

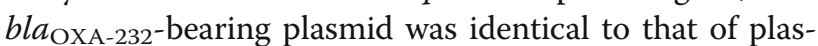
mid pKP3-A bearing the $b l a_{\mathrm{OXA}-181}$ gene [20]. The $b l a_{\text {OXA-232 }}$ gene was part of a truncated Tn2013 transposon. Disrupting the ISEcp1 transposase activity, the deletion may have stabilized the $b l a_{\mathrm{OXA}-232}$ gene on the ColE-like plasmid [9]. The high nucleotide sequence

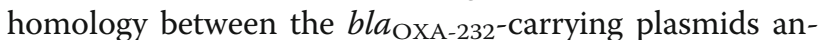
alyzed in this study and the previously reported plasmid sequences revealed possible sources of the plasmid, and also indicated the significant role of the ColKP3 plasmids on the prevalence of $b l a_{\mathrm{OXA}-232}$ gene in China.

\section{Conclusion}

A clonal dissemination and outbreak of OXA232Kp has been observed, and $K$. pneumoniae ST15 was the predominant clone. Most OXA232Kp strains exhibited lowlevel resistance to carbapenems. All strains carry a 6141 bp ColKP3 plasmid harboring the bla $a_{\text {OXA-232 }}$ gene, which is highly homologous to other bla $a_{\text {OXA-232-bearing }}$ plasmids involved in other studies from the same region.

\section{Abbreviations}

AK: amikacin; ATCC: American Type Culture Collection; CAZ: Ceftazidime; CIP: Ciprofloxacin; CLSI: Clinical Laboratory Standards Institute; CTX: Cefotaxime; ETP: Ertapenem; ICU: Intensive Care Unit; IMP: Imipenem; MEM: Meropenem; MS: Mass spectrum; OXA232Kp: OXA-232-producing K. pneumoniae; PB: Polymyxin B; PFGE: Pulsed-field gel electrophoresis; ST: Sequence type; SXT: Sulfamethoxazole/trimethoprim; TGC: Tigecycline; TZP: Piperacillin-tazobactam

\section{Acknowledgements}

Not applicable.

\section{Consent to publish}

Not applicable.

\section{Authors' contributions}

$\mathrm{SL}, J Y$ and $B L$ designed the study. $\mathrm{XL}, \mathrm{WM}$, and $\mathrm{LY}$ did phenotypic and genotypic analysis. WM and QQ participated in the collection of strains. JY drafted the manuscript. All authors read and approved the final manuscript. All authors declare that they have no conflicts of interest.

\section{Funding}

This study was supported by the Special Key Project of Biosafety Technologies for the National Major Research \& Development Program of China (2017YFC1200803). The funding provides research expenditure for this study to purchase reagents and experimental consumables for related experiments.

Availability of data and materials

All the sequences were uploaded to GenBank with BioProject Accession PRJNA523565. All data generated or analysed during this study are included in this published article.

Ethics approval and consent to participate

No ethical approval was obtained for using the clinical samples since they were collected during routine bacteriologic analyses in public hospitals. All data were anonymously analyzed.

\section{Competing interests}

The authors have no conflicts of interest to declare.

\section{Author details}

${ }^{1}$ Center for Clinical Laboratory Medicine, Chinese PLA General Hospital, Beijing 100853, China. ${ }^{2}$ Department of Laboratory Diagnosis, Changhai Hospital, Navy Medical University, Shanghai 200433, China. ${ }^{3}$ Center for Clinical Laboratory, the 302 Hospital of Chinese PLA, Beijing 100039, China.

Received: 17 March 2019 Accepted: 9 October 2019

Published online: 28 October 2019

References

1. Logan LK, Weinstein RA. The Epidemiology of Carbapenem-Resistant Enterobacteriaceae: The Impact and Evolution of a Global Menace. J Infect Dis. 2017;215(suppl_1):S28-36. 
2. Poirel $L$, Heritier $C$, Tolun V, Nordmann P. Emergence of oxacillinasemediated resistance to imipenem in Klebsiella pneumoniae. Antimicrob Agents Chemother. 2004;48(1):15-22.

3. Mairi A, Pantel A, Sotto A, Lavigne JP, Touati A. OXA-48-like carbapenemases producing Enterobacteriaceae in different niches. Eur J Clin Microbiol Infect Dis. 2018;37(4):587-604.

4. Tafoukt R, Leangapichart T, Hadjadj L, Bakour S, Diene SM, Rolain JM, Touati A. Characterisation of blaOXA-538, a new variant of blaOXA-48, in Shewanella xiamenensis isolated from river water in Algeria. J Glob Antimicrob Resist. 2018;13:70-3.

5. Poirel L, Potron A, Nordmann P. OXA-48-like carbapenemases: the phantom menace. J Antimicrob Chemother. 2012;67(7):1597-606

6. Ma L, Wang JT, Wu TL, Siu LK, Chuang YC, Lin JC, Lu MC, Lu PL. Emergence of OXA-48-producing Klebsiella pneumoniae in Taiwan. PLoS One. 2015; 10(9):e0139152.

7. Guo L, An J, Ma Y, Ye L, Luo Y, Tao C, Yang J. Nosocomial outbreak of OXA48-producing Klebsiella pneumoniae in a Chinese hospital: clonal transmission of ST147 and ST383. PLOS One. 2016;11(8):e0160754.

8. Yu F, Wang S, LV J, Qi X, Guo Y, Tang YW, Kreiswirth BN, Wang L, Chen L. Coexistence of OXA-48-producing Klebsiella pneumoniae and Escherichia coli in a hospitalized patient who returned from Europe to China. Antimicrob Agents Chemother. 2017;61(4):e02580-16.

9. Potron A, Rondinaud E, Poirel L, Belmonte O, Boyer S, Camiade S, Nordmann P. Genetic and biochemical characterisation of OXA-232, a carbapenem-hydrolysing class D beta-lactamase from Enterobacteriaceae. Int J Antimicrob Agents. 2013;41(4):325-9.

10. Al-Marzooq F, Ngeow YF, Tay ST. Emergence of Klebsiella pneumoniae producing dual carbapenemases (NDM-1 and OXA-232) and 16S rRNA methylase (armA) isolated from a Malaysian patient returning from India. Int J Antimicrob Agents. 2015;45(4):445-6.

11. Doi Y, O'Hara JA, Lando JF, Querry AM, Townsend BM, Pasculle AW, Muto CA. Co-production of NDM-1 and OXA-232 by Klebsiella pneumoniae. Emerg Infect Dis. 2014;20(1):163-5.

12. Jeong SH, Lee KM, Lee J, Bae IK, Kim JS, Kim HS, Song W. Clonal and horizontal spread of the blaOXA-232 gene among Enterobacteriaceae in a Korean hospital. Diagn Microbiol Infect Dis. 2015;82(1):70-2.

13. Torres-Gonzalez P, Cervera-Hernandez ME, Niembro-Ortega MD, Leal-Vega F, Cruz-Hervert LP, Garcia-Garcia L, Galindo-Fraga A, Martinez-Gamboa A Bobadilla-Del Valle M, Sifuentes-Osornio J, et al. Factors associated to prevalence and incidence of Carbapenem-resistant Enterobacteriaceae fecal carriage: a cohort study in a Mexican tertiary care hospital. PLoS One. 2015; 10(10):e0139883.

14. Abdul Momin MHF, Liakopoulos A, Phee LM, Wareham DW. Emergence and nosocomial spread of carbapenem-resistant OXA-232-producing Klebsiella pneumoniae in Brunei Darussalam. J Glob Antimicrob Resist. 2017;9:96-9.

15. Laolerd W, Akeda Y, Preeyanon L, Ratthawongjirakul P, Santanirand P. Carbapenemase-producing Carbapenem-resistant Enterobacteriaceae from Bangkok, Thailand, and their detection by the Carba NP and modified Carbapenem inactivation method tests. Microb Drug Resist. 2018;24(7):1006-11.

16. Avolio M, Vignaroli C, Crapis M, Camporese A. Co-production of NDM-1 and OXA-232 by ST16 Klebsiella pneumoniae, Italy, 2016. Future Microbiol. 2017; 12:1119-22.

17. Mancini S, Poirel L, Tritten ML, Lienhard R, Bassi C, Nordmann P. Emergence of an MDR Klebsiella pneumoniae ST231 producing OXA-232 and RmtF in Switzerland. J Antimicrob Chemother. 2018:73(3):821-3.

18. Findlay J, Hopkins KL, Loy R, Doumith M, Meunier D, Hill R, Pike R, Mustafa $\mathrm{N}$, Livermore DM, Woodford N. OXA-48-like carbapenemases in the UK: an analysis of isolates and cases from 2007 to 2014. J Antimicrob Chemother. 2017;72(5):1340-9.

19. Espinal P, Nucleo E, Caltagirone M, Mattioni Marchetti V, Fernandes MR, Biscaro V, Rigoli R, Carattoli A, Migliavacca R, Villa L: Genomics of Klebsiella pneumoniae ST16 producing NDM-1, CTX-M-15, and OXA-232. Clin Microbiol Infect 2019, 25(3):385.e1-385.e5.

20. Potron A, Nordmann P, Lafeuille E, Al Maskari Z, Al Rashdi F, Poirel L. Characterization of OXA-181, a carbapenem-hydrolyzing class D betalactamase from Klebsiella pneumoniae. Antimicrob Agents Chemother. 2011;55(10):4896-9.

21. Yin D, Dong D, Li K, Zhang L, Liang J, Yang Y, Wu N, Bao Y, Wang C, Hu F. Clonal dissemination of OXA-232 Carbapenemase-producing Klebsiella pneumoniae in neonates. Antimicrob Agents Chemother. 2017:61(8): e00385-17.
22. Tian D, Pan F, Wang C, Sun Y, Zhang H. Resistance phenotype and clinical molecular epidemiology of carbapenem-resistant Klebsiella pneumoniae among pediatric patients in Shanghai. Infect Drug Resist. 2018;11:1935-43.

23. Shu L, Dong N, Lu J, Zheng Z, Hu J, Zeng W, Sun Q, Wai-Chi Chan E, Zhou $\mathrm{H}, \mathrm{Hu} \mathrm{F}$, et al. Emergence of OXA-232 carbapenemase-producing Klebsiella pneumoniae that carries a pLVPK-like virulence plasmid among elderly patients in China. Antimicrob Agents Chemother. 2019;63(3):e02246-18.

24. Poirel L, Walsh TR, Cuvillier V, Nordmann P. Multiplex PCR for detection of acquired carbapenemase genes. Diagn Microbiol Infect Dis. 2011;70(1):119-23.

25. Solgi H, Badmasti F, Giske CG, Aghamohammad S, Shahcheraghi F. Molecular epidemiology of NDM-1- and OXA-48-producing Klebsiella pneumoniae in an Iranian hospital: clonal dissemination of ST11 and ST893. J Antimicrob Chemother. 2018;73(6):1517-24.

26. Yang J, Ye L, Guo L, Zhao Q, Chen R, Luo Y, Chen Y, Tian S, Zhao J, Shen D, et al. A nosocomial outbreak of KPC-2-producing Klebsiella pneumoniae in a Chinese hospital: dissemination of ST11 and emergence of ST37, ST392 and ST395. Clin Microbiol Infect. 2013;19(11):E509-15.

27. Tenover FC, Arbeit RD, Goering RV, Mickelsen PA, Murray BE, Persing DH, Swaminathan B. Interpreting chromosomal DNA restriction patterns produced by pulsed-field gel electrophoresis: criteria for bacterial strain typing. J Clin Microbiol. 1995;33(9):2233-9.

28. Thomas CP, Moore LS, Elamin N, Doumith M, Zhang J, Maharjan S, Warner M, Perry C, Turton JF, Johnstone C, et al. Early (2008-2010) hospital outbreak of Klebsiella pneumoniae producing OXA-48 carbapenemase in the UK. Int J Antimicrob Agents. 2013;42(6):531-6.

29. Lascols C, Peirano G, Hackel M, Laupland KB, Pitout JD. Surveillance and molecular epidemiology of Klebsiella pneumoniae isolates that produce carbapenemases: first report of OXA-48-like enzymes in North America. Antimicrob Agents Chemother. 2013;57(1):130-6.

30. Arana DM, Saez D, Garcia-Hierro P, Bautista V, Fernandez-Romero S, Angel De La Cal M, Alos JI, Oteo J. Concurrent interspecies and clonal dissemination of OXA-48 carbapenemase. Clin Microbiol Infect. 2015;21(2):148.e1-4.

31. Daikos GL, Markogiannakis A. Carbapenemase-producing Klebsiella pneumoniae: (when) might we still consider treating with carbapenems? Clin Microbiol Infect. 2011;17(8):1135-41.

\section{Publisher's Note}

Springer Nature remains neutral with regard to jurisdictional claims in published maps and institutional affiliations.

\section{Ready to submit your research? Choose BMC and benefit from:}

- fast, convenient online submission

- thorough peer review by experienced researchers in your field

- rapid publication on acceptance

- support for research data, including large and complex data types

- gold Open Access which fosters wider collaboration and increased citations

- maximum visibility for your research: over $100 \mathrm{M}$ website views per year

At $\mathrm{BMC}$, research is always in progress.

Learn more biomedcentral.com/submissions 